\title{
Polypoidal Metastatic Leiomyosarcoma Diffusely Involving the Gastrointestinal Tract
}

\author{
Anuradha Sekaran ${ }^{1}$, Supraja Sukumar ${ }^{1}$, Rohini Muriki ${ }^{2}$, Rakesh Kalapala $^{2}$, Sundeep Lakhtakia ${ }^{2}$, \\ Duvvur Nageshwar Reddy ${ }^{2}$
}

Departments of ${ }^{1}$ Pathology and ${ }^{2}$ Medical Gastroenterology, AIG Hospitals, Hyderabad, Telangana, India.

\section{Corresponding Author:}

Dr. Anuradha Sekaran

Email: dr.sanuradha@aighospitals.com

This is an Open Access article distributed under the terms of the Creative Commons Attribution License (creativecommons.org/ licenses/by/3.0).

Received

Accepted

Published

July 28,2020

November 24, 2020

December 15, 2020

\begin{abstract}
Background: Retroperitoneal leiomyosarcoma, a malignant neoplasm with smooth muscle differentiation, seldom metastasize. The gastrointestinal tract is rare site of metastases, and diffuse polypoidal involvement is an uncommon presentation. Case Report: A 61-year-old male diagnosed with retroperitoneal leiomyosarcoma underwent surgical resection of the tumor along with chemotherapy and radiation. He presented with 2 months history of vomiting, and melena. Upper gastrointestinal (GI) endoscopy, capsule endoscopy and colonoscopy revealed numerous polypoidal lesions throughout the gastrointestinal tract, suspicious of a GI polyposis syndrome. Histopathology, including immunohistochemistry, confirmed metastatic leiomyosarcoma. Conclusion: Diffuse polypoidal lesions in the gastrointestinal tract presenting as metastases from a retroperitoneal leiomyosarcoma are extremely rare.
\end{abstract}

Keywords: Colonoscopy, Endoscopy, Gastrointestinal Neoplasms, Melena, Retroperitoneal Leiomyosarcomas.

\section{Introduction}

Leiomyosarcoma(LMS), together with liposarcoma account for $5-10 \%$ of all soft tissue sarcomas [1,2]. LMS is a malignant neoplasm of smooth muscle origin that can arise from peripheral soft tissues, genitourinary tract, gastrointestinal tract, large vessels and even in bones. The management and prognosis differ according to the location of primary tumor. The usual sites of metastases are the liver and lungs [3]. Metastasis to the gastrointestinal tract (GIT) from a primary retroperitoneal site is unusual. Here we report a case of retroperitoneal leiomyosarcoma with diffuse metastases to the GIT having a polypoidal appearance.

\section{Case Report}

A 61-year-old male underwent surgical resection of a large $(11.4 \times 8.5 \times 9.2 \mathrm{~cm})$ retroperitoneal soft tissue mass 3 years ago. Histopathology showed fascicles of spindled cells with nuclear hyperchromasia and pleomorphism with a mitotic index of 10-15/10 hpf. On immunohistochemistry (IHC), smooth muscle actin (SMA) and desmin were positive, while CD-117, DOG-1, CD-99, Bcl-2 and S-100 were negative, confirming the diagnosis of leiomyosarcoma grade 2 [Fédération nationale des centres de lute contre le cancer (FNCLCC)]. He received 4 cycles of chemotherapy (iphosphamide and adriamycin) prior to surgery. Post-surgery, he received 2 cycles (gemcitabine and docetaxel) followed by IMRT (intensity modulated radiotherapy), in view of the large size and the positive surgical margins. The total radiation dose given under image guidance was $54 \mathrm{~Gy}$ in 27 fractions. He tolerated the treatment well.

He presented now with recurrent vomiting, excessive belching, hiccups and intermittent 
melena for the past 2 months. Investigations revealed severe anemia (hemoglobin: $6.8 \mathrm{gm} \%$ ), mild hypoalbuminemia, hyponatremia and elevated INR (2.88). Upper gastrointestinal endoscopy (UGIE) revealed multiple polyps in the oesophagus $(0.5 \times 0.5 \mathrm{~cm})$, body and fundus of stomach (largest $1 \times 1 \mathrm{~cm}$ with ulceration) and large wide based polyps in first and second part of duodenum $(3 \times 3 \mathrm{~cm})$ having irregular surface [Fig.1]. Capsule endoscopy showed multiple duodenal, jejunal and ileal polyps, jejunal angioectasia with erosions and proximal ileal ulcer with stricture and causing near total luminal occlusion [Fig.2]. Colonoscopy revealed 2 pedunculated polyps in the sigmoid colon [Fig.3] and a single large polyp measuring $3 \mathrm{~cm}$ in maximum dimension in the transverse colon [Fig.3-Inset]. Polypectomy of the largest gastric polyp, duodenal polyp and transverse colon polyp was performed. Histopathology of all the polyps showed a similar appearance of tumor, infiltrating the glands, crypts and villi, composed of elongated spindled cells with eosinophilic cytoplasm and plump cigar-shaped vesicular nucleus [Fig.4]. The tumor cells displayed marked nuclear pleomorphism and occasional bizarre cells. Mitosis was around 7-8/10 hpf with atypical mitotic figures, without any appreciable necrosis [Fig.5]. Immunohistochemistry showed diffuse cytoplasmic positivity for SMA [Fig.6], desmin and caldesmon; immunonegative for CD-117, DOG1 and S-100 excluding gastrointestinal stromal tumor (GIST) and schwannoma, respectively as differential diagnoses. The Ki67 proliferative index was 30\% [Fig.6-Inset], thus confirming our diagnosis of a metastatic leiomyosarcoma, grade 1 (FNCLCC) and involving stomach, duodenum and colon.

The patient underwent best palliative care in our hospital and was discharged.

\section{Discussion}

Leiomyosarcomas can occur in any anatomical site. The clinical outcome depends on the site of origin.

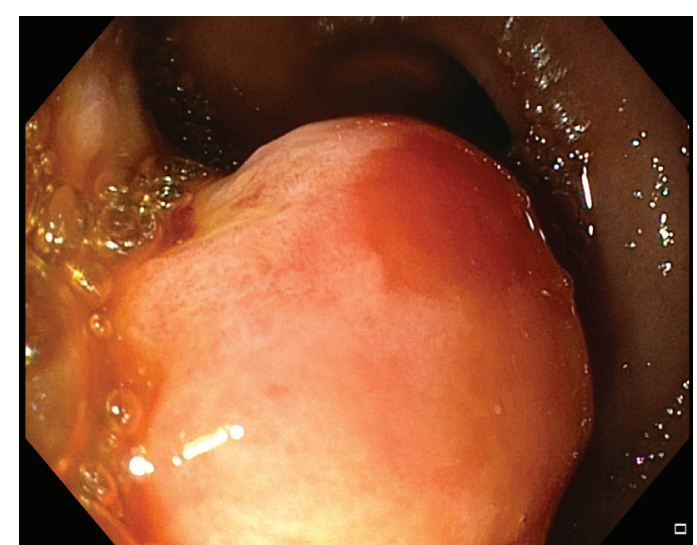

Fig.1: Upper gastrointestinal endoscopy: Gastric polyp obstructing lumen.

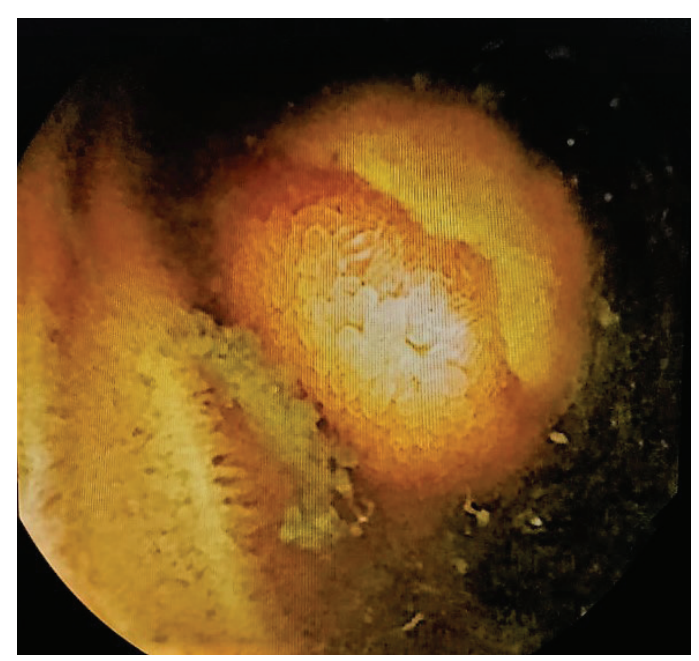

Fig.2: Capsule endoscopy: Polyp at proximal ileum with focal ulceration.

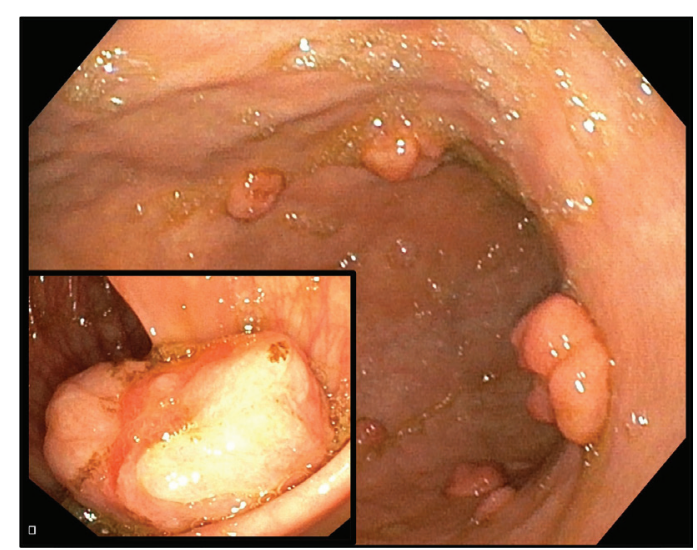

Fig.3: Colonoscopy: Multiple polyps throughout the colon with inset showing a large polyp. 


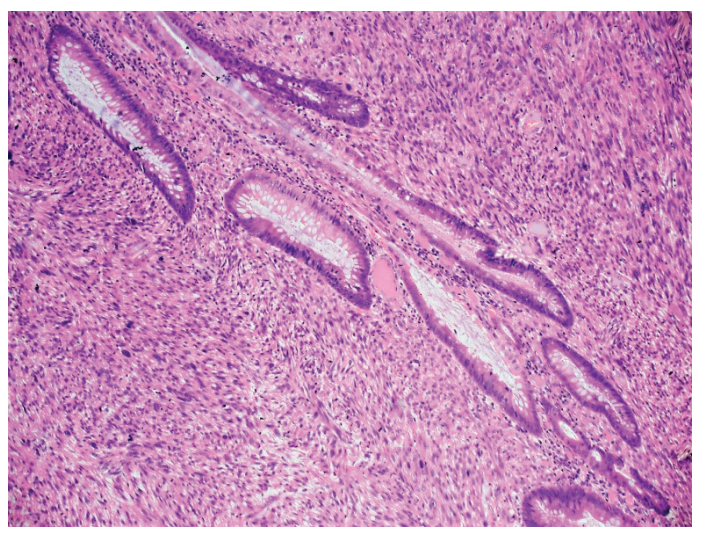

Fig.4: Spindled tumor cells infiltrating between the crypts, $H \& E 10 \times$.

The most common site is the retroperitoneum. It typically affects middle-aged to older adults [4]. Diagnosis is delayed because most patients are asymptomatic. Cure of the primary tumor is often not possible due to late presentation, location in deep tissue, inability to achieve wide surgical margins, and relative insensitivity to chemotherapy and radiotherapy. These tumors have propensity for hematogenous spread and infrequently metastasize to lymph nodes. Distant metastases may be observed at the time of diagnosis in approximately $40 \%$ of cases. Most patients who survive the primary tumor eventually develop distant metastases and hence associated with poor prognosis $[5,6]$.

Our patient presented with widespread metastases in the gastrointestinal tract (oesophagus, stomach, duodenum and other parts of small bowel, and colon) about after 3 years post-surgery and chemo-radiotherapy. Diffuse polypoidal involvement of the gastrointestinal tract (from the oesophagus to the transverse colon) has not been reported in literature, although isolated involvement to the stomach has been reported [7]. The clinical features may be non-specific (such as dyspepsia, belching), to more organic symptoms e.g. abdominal pain, recurrent vomiting caused by obstruction, recurrent bleeding leading to anemia, or even acute abdomen due to perforation, depending on the location of the metastasis $[8,9]$.

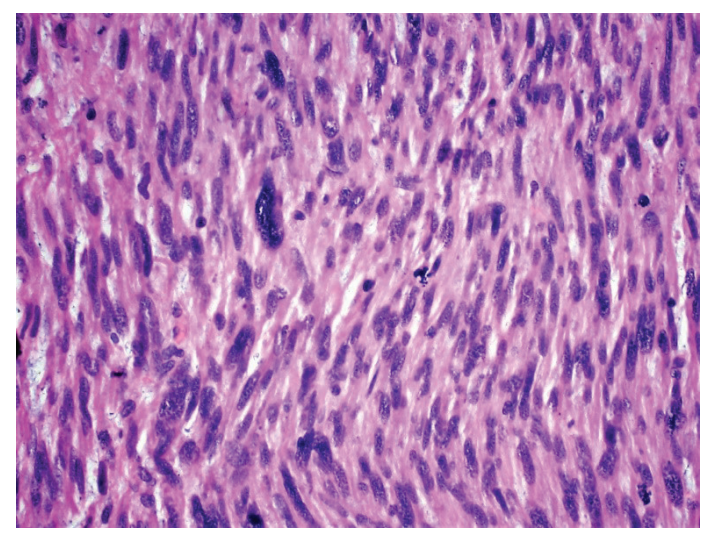

Fig.5: The tumor cells displayed marked nuclear pleomorphism and occasional bizarre cells, $H \& E 40 \times$.

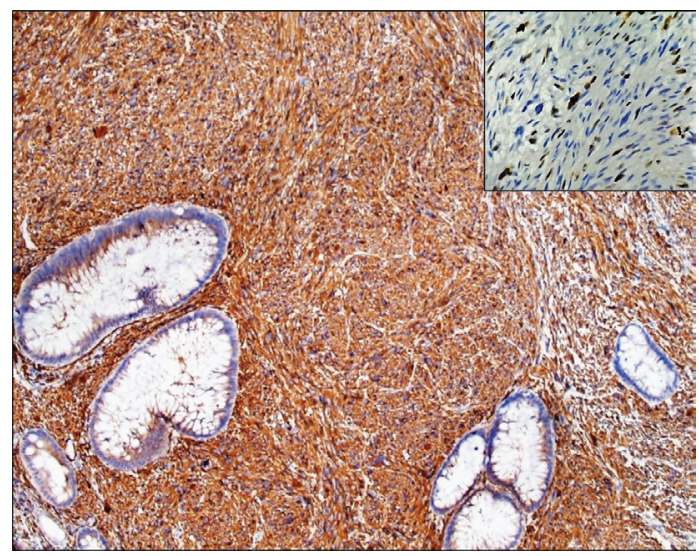

Fig.6: Immunohistochemistry (IHC) showed diffuse cytoplasmic positivity for smooth muscle actin SMA, 10× (Inset- Ki-67 proliferative index was 30\%, 40×).

The index case had extensive evaluation including UGIE, colonoscopy and capsule endoscopy that revealed multiple polypoidal lesion spread over various segments of GIT. The common differential diagnoses of spindle cell lesions on histopathological morphology are GIST and leiomyosarcoma. However, the immunohistochemical profile being negative for CD-117 and DOG-1 did not favour GIST. Also, GIST does not present with widespread GI mucosal metastases.

In this case, the features suggestive of metastatic LMS (over a primary LMS of the 
GIT) include supporting history, simultaneous development of multiple polyps, multi-segmental involvement, rapid growth, involvement of the stomach (unusual for LMS) and the location of the tumor in the submucosa with secondary ulceration of the mucosa.

\section{Conclusion}

This case illustrates an unusual pattern of metastases of a leiomyosarcoma originating from the retroperitoneum to the gastrointestinal tract having diffuse involvement as multiple polypoidal tumors. Being a high-grade, therapyresistant neoplasm with poor prognosis, awareness of atypical metastatic sites is essential for early diagnosis.

Acknowledgement: We would like to acknowledge our technical staff (Mr. Gopal Rajput, Prashant Bonige and Manga Chanchati) for their support.

Contributors: AS: conception of study, data analysis, interpretation and critical revision; SS: data collection, analysis; RM: data collection; RK, DRN: review of manuscript; SL: critical revision of manuscript. AS will act as a study guarantor. All authors approved the final version of this manuscript and are responsible for all aspects of this study.

Funding: None; Competing interests: None stated.

\section{References}

1. Cancer Trends Progress Report National Cancer Institute, NIH, DHHS, Bethesda, MD, March 2020. Available at: https://progressreport.cancer.gov/ Accessed on July 27, 2020.

2. Singh Z. Leiomyosarcoma: A rare soft tissue cancer arising from multiple organs. Journal of Cancer Research and Practice. 2018;5(1):1-8.

3. Marko J, Wolfman DJ. Retroperitoneal leiomyosarcoma from the Radiologic Pathology Archives. Radiographics. 2018;38(5):1403-1420.

4. Lou J, Moten AS, Reddy SS, Howell KJ, Movva S, von Mehren M, et al. Retroperitoneal sarcomas: Does laterality matter? J Surg Res. 2019;244:34-41.

5. Li Q, Zhuang R, Zhu J, Lu W, Hou Y, Liu J, et al. Prognostic factors in patients with recurrent or metastatic retroperitoneal leiomyosarcoma. Future Oncol. 2015;11(12):1759-1766.

6. Puie N, Trică LP, Nagy GA, Rusu I, Şovrea AS, Georgiu C. Hemorrhagic polypoid gastric and colonic metastases nine years after uterine leiomyosarcoma - case report. Rom J Morphol Embryol. 2017;58(3):1017-1022.

7. Dent LL, Cardona CY, Buchholz MC, Peebles R, Scott JD, Beech DJ, et al. Soft tissue sarcoma with metastasis to the stomach: a case report. World J Gastroenterol. 2010;16(40):5130-5134.

8. Oda I, Kondo H, Yamao T, Saito D, Ono H, Gotoda T, et al. Metastatic tumors to the stomach: analysis of 54 patients diagnosed at endoscopy and 347 autopsy cases. Endoscopy. 2001;33(6):507-510.

9. Galanopoulos M, Gkeros F, Liatsos C, Pontas C, Papaefthymiou A, Viazis N, et al. Secondary metastatic lesions to colon and rectum. Ann Gastroenterol. 2018;31(3):282-287. 